\title{
Effect of local anti-vascular endothelial growth factor therapy to prevent the formation of stenosis in outflow vein in arteriovenous fistula
}

\author{
Xin Huang ${ }^{1}$, Jibin Guan², Zitong Sheng ${ }^{1}$, Menghua Wang ${ }^{1}$, Tianhua Xu' ${ }^{1}$, Guangying \\ Guo $^{1}$, Pengzhi Wan ${ }^{1}$, Binyao Tian ${ }^{1}$, Junlei Zhou ${ }^{1}$, Aoran Huang ${ }^{1}$, Junfeng Hao ${ }^{3}$, Li Yao ${ }^{1}$ \\ 'Department of Nephrology, The First Hospital of China Medical University, Shenyang 110001, Liaoning \\ Province, China. \\ ${ }^{2}$ College of Pharmacy, University of Minnesota, Minneapolis 55455, MN, USA. \\ ${ }^{3}$ Department of Nephrology, Jinqiu Hospital Liaoning Province, Shenyang 110016, Liaoning Province, China
}

Address for Correspondence:

Li Yao, Department of Nephrology, The First Hospital of China Medical University, No. 92 Nanjing Street, Heping District, Shenyang 110001, Liaoning Province, China.E-mail: liyao_cmu@163.com;

Junfeng Hao, Department of Nephrology, Jingiu Hospital Liaoning Province, No. 317 Xiaonan Street, Shenhe District, Shenyang 110016, Liaoning Province, China. E-mail: ygzhif@163.com

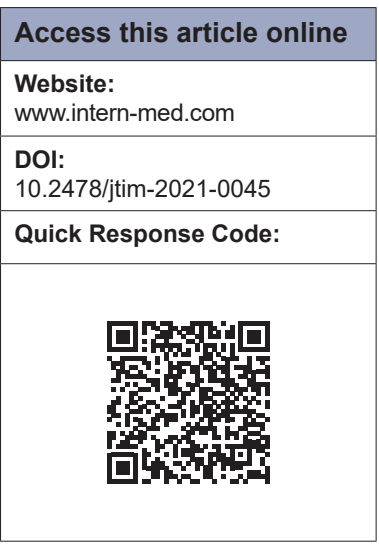

\section{ABSTRACT}

Background and Objectives: Vascular stenosis and angiogenesis are the major causes of short expectancy of arteriovenous fistula (AVF). Increased expression of vascular endothelial growth factor-A (VEGF-A) has been suggested to play an important role in the pathophysiologic process. Anti-VEGF has been proved to be effective on anti-angiogenesis and applied in clinical practice, but its effect on anti-stenosis remains to be verified before it could be applied to prevent stenosis of AVF. This study was aimed to evaluate the effect of local anti-VEGF therapy to prevent the formation of stenosis in the outflow vein in AVF and its mechanism. Methods: Bioinformatics of VEGF-A and its downstream-regulated molecules from the STRING PPI database were analyzed in this study. The biopsy samples from outflow veins of AVF in patients and C57BL/6 mouse models were analyzed to examine the mechanisms of pathologic vascular stenosis associated with VEGF pathways and their potential therapeutic targets. Results: We found that the reduction of VEGF-A could downregulate downstream molecules and subsequently reduce the intimal hyperplasia and abnormal vascular remodeling by analyzing the STRING PPI database. Venous wall thickening, intimal neointima formation, and apoptosis of vascular endothelial cells in the proliferative outflow vein of the AVF were significantly more obvious, and upregulation of expression of VEGF was observed in dysfunctional AVF in patients. In mouse models, the expression of VEGF, Ephrin receptor B4 (EphB4), matrix metalloproteinase (MMP)2, MMP9, tissue inhibitor of metalloproteinase (TIMP)1, TIMP2, and caspase 3 in the control-shRNA surgical group was significantly higher than in the sham group $(P<0.05)$, and all of these indicators were significantly lower in lentiviral transfection group and Avastin group than in control-shRNA surgical group $(P<0.05)$ on the 14th day after AVF operation. Conclusion: VEGF expression is significantly increased in vascular endothelial cells in stenosed or occluded outflow veins of dysfunctional AVF. Local injection of Avastin into the adventitia of the proximal outflow vein in autologous AVF procedure has an excellent potential to prevent the subsequent local stenosis of the proximal outflow vein.

Key words: arteriovenous fistulas, angiogenesis, vascular endothelial growth factor A, Avastin

\section{INTRODUCTION}

Vascular access is the lifeline of patients with an end-stage renal disease (ESRD) requiring hemodialysis (HD). Autologous arteriovenous fistula (AVF) is considered the preferred choice for vascular access in HD. ${ }^{[1]}$ However, the utility of AVFs suffers from a high failure ratio, and its 1-year primary patency rate is estimated to be only 
$60 \%-65 \%{ }^{[2,3]}$ The cost of vascular access operation, in addition to that of HD treatment, imposes a heavy financial burden on patients. According to the United States Renal Data System (USRDS) report, the total expenses of HD are estimated at $\$ 90,971$ per patient per year in the USA. ${ }^{[4]}$ The exact pathophysiology is not yet clear. We presumed that outflow vein stenosis, as a vital cause of malfunction of AVF, is mainly attributed to the following two factors: an initial inability to mature and the later development of stenotic lesions in the outflow vein triggered by local ischemia and oxidative stress. The mechanism of intimal hyperplasia includes the upstream and downstream cascade events. Upstream events mainly include endothelial and smooth muscle injury caused by various factors, including local vascular damage during AVF surgery, hemodynamic changes (especially the increase in vascular shear force) after the formation of AVF, and repeated puncture in the process of subsequent use. These factors, in turn, trigger the interaction of cells, cytokines, and mediators, leading to the proliferation of neointima and interacting with the injury of uremia to endothelial cells (ECs) to accelerate venous neointima proliferation. ${ }^{[5,6]}$ Downstream events consist of a series of pathophysiologic responses to the previous endothelial (vascular) injury, including oxidative stress, inflammation, and EC dysfunction. ${ }^{[7,8]}$ Caused by the upstream events mentioned above, the downstream ones lead to smooth muscle cell migration from the middle membrane to the intima hyperplasia, leading to intimal hyperplasia. ${ }^{[9]}$ Therefore, it is crucial to clarify the mechanisms of the venous stenosis formation and, based on this, develop strategies to prevent or delay the failure of AVF and graft.

The pathophysiologic changes of neoendothelial hyperplasia $(\mathrm{NH})$ involve several types of cells, including ECs, vascular smooth muscle cells (VSMCs), and adventitial fibroblasts (AFs). ${ }^{[10]}$ AFs are the precursor cells of myofibroblasts ( $\alpha$-SMA-positive cells found in specimens removed from patients with failed AVFs), ${ }^{[1]}$ which promote the formation of venous neointimal hyperplasia $(\mathrm{VNH})^{[12,13]}$ in the subsequent process. This process of injury is orchestrated by a large number of mediators such as the growth factors vascular endothelial growth factor (VEGF), ${ }^{[14,15]}$ platelet-derived growth factor (PDGF), ${ }^{[16]}$ insulin-like growth factor-1 (IGF-1), ${ }^{[17]}$ and basic fibroblast growth factor (bFGF). ${ }^{[18]}$ Therefore, effective treatment must ensure that the concentration of the drug is sufficient to block the cascade reaction of venous stenosis formation effectively. We focused on one of the most potent angiogenic factors, VEGF-A, which is involved in the pathogenesis of stenosis of artery/vein bypass grafts and $\mathrm{VNH}$ associated with dysfunction of $\mathrm{HD}$ vascular access. ${ }^{[19-21]}$ Previous work has demonstrated that the expression of VEGF-A, VEGF receptor (VEGFR)-1, and
VEGFR-2 increased at the site of venous stenosis in lesions of AVF and graft failure in murine and porcine models. Other researches suggested that matrix metalloproteinase (MMP)2 and MMP9 also participated in the process of AVF stenosis. ${ }^{[22,23]}$ We predicted the bioinformatics network of interaction among VEGF-A and its related regulated MMP2, tissue inhibitor of metalloproteinase 1 (TIMP1), TIMP2 and Ephrin receptor B4 (EphB4) molecules by analyzing the STRING PPI database. As a humanized monoclonal antibody to VEGF-A, Avastin (Bevacizumab; Genentech, San Francisco, CA, USA) has been proved effective on anti-angiogenesis and applied in clinical practice. But its effect on anti-stenosis remains to be verified before it could be applied to prevent stenosis of AVF in clinical practice. In this study, bioinformatics analysis studies and animal experiments are designed to investigate whether it would decrease VNH formation. Furthermore, a small hairpin lentivirus (LV)-RNA (shRNA)-VEGF-A (LV-shRNA-VEGF-A) and Avastin were injected into the adventitial of outflow vein of the AVF in murine models in different groups, respectively. Specimens extracted from intraoperatively resected tissue were also analyzed to verify if the stenosis of the outflow vein after end-to-side anastomotic AVF during postoperative use stems from very similar pathophysiologic processes.

\section{METHODS}

\section{Bioinformatics analysis}

Bioinformatics of VEGF-A and its downstream-regulated MMP2, TIMP1, TIMP2 and EphB4 molecules was analyzed from STRING PPI database (https://string-db. org).

\section{Patients}

Specimens were collected from 20 of the 121 patients undergoing AVF surgery over 3 months (between January 1 and March 31) at the Hemodialysis Unit, First Affiliated Hospital of China Medical University (Shenyang, China). All subjects had signed informed consent before undergoing the operations for retention of the specimen excised for research purposes in this study. The study was approved by the institutional review board (Ethical Lot Number: AF-SOP-07-1,1-01).

All the patients underwent preoperative clinical assessment with ultrasound imaging by a vascular sonographer who measured the vessel size and other variables (including distensibility, vessel wall thickness, and resistance index) to evaluate the suitability of vessels for AVF access creation or re-anastomosis surgery. ${ }^{[24]}$ Patients with active inflammatory diseases or neoplasia or those receiving immunosuppressive therapy were excluded. 
Thirty-one of the 121 patients underwent surgical repair of clinically significant stenosis or thrombosed AVF. Clinically significant stenosis is defined as the inability to achieve the prescribed dialysis blood pump flow rate in at least two consecutive HD sessions and any one of the following criteria on ultrasound: (1) AVF with blood flow rate $<400 \mathrm{~mL} / \mathrm{min}$, (2) a ratio of venous access pressure to main arterial pressure $>0.55$, (3) peak systolic velocities of $\geq 500 \mathrm{~cm} / \mathrm{sec}$, and (4) $\geq 50 \%$ reduction in luminal diameter in comparison with the adjacent vessel. ${ }^{[25]}$ Twenty of the patients were diagnosed with brachiocephalic AVF (B-C AVF) primary stenosis, and 16 of them had used the AVF for more than 2 years without any interventional treatment. Thirteen of the 16 patients were diagnosed with apparent intimal hyperplasia defined as juxta-anastomotic intimalmedial thickness (IMT) $>50 \%$ on ultrasound. IMT was determined as a low-level echo-gray band (not projecting into the vessel lumen) and was measured by the following formula:

IMT $\%=$ (outer to outer vessel wall diameter - luminal diameter)/outer to outer wall vessel diameter $\times 100 \% .{ }^{[26,27]}$

Ten of the 13 patients were included in the case group. A radiocephalic AVF was revised by the formation of a neo-anastomosis in the proximal cephalic vein segment in each patient of the case group. A 1-5-mm-long specimen of the stenosis of the vein stump was taken from each of the 10 patients included in the study. The first 10 patients undergoing end-vein-to-side-artery anastomotic B-C AVF creation with specimens obtained during the operation were included in the control group. A 1-2-mm-long specimen of the normal vein was taken from each of the 10 patients included in the study.

All specimens were obtained from the pruning process of the venous anastomotic site, and no venous tissue samples were obtained from other normal vessels or locations in the field. The stenosis was confirmed by histopathology. None of the stenosis or neointimal hyperplasia lesions included in this study had been treated by percutaneous transluminal angioplasty (PTA) before the surgical resection. No patient had performed a phlebography.

\section{Animals}

A total of 48 male C57BL/6 mice (Charles River, Wilmington, MA, USA) (Ethical Lot Number: CMU2021201) weighing 25-30 g and aged 8-10 weeks were randomly divided into four groups $(n=12$, respectively): sham group(sham), control-shRNA group (nontargeting empty vector, scrambled-shRNA injected locally), LV-shRNA-VEGF-A group (LV-shRNAVEGF-A injected locally), and Avastin group (Avastin injected locally). Renal function was determined in all the
48 mice by measuring serum urea nitrogen and creatinine levels at baseline and 4 weeks after adenine feeding. A unilateral AVF was established between the dorsal branch of the external jugular vein and the common carotid artery in the three groups on day 0 . Either $1 \times 10^{6}$ particleforming units (PFU) of LV-shRNA-VEGF-A or controlshRNA-VEGF-A were transduced into the adventitia of the proximal outflow vein at the time of AVF creation, depending on the group. Avastin $(5 \mathrm{mg} / \mathrm{kg})$ in $10 \mu \mathrm{L}$ of phosphate-buffered saline (PBS) was injected locally into the adventitia of the proximal outflow vein using a $30 \mathrm{G}$ needle during operation. The injection region of proximal outflow veins was harvested after euthanasia on day14 for further study.

\section{Tissue harvesting and processing}

At the time of the endpoint, all mice were anesthetized as described previously before the outflow veins were freed and dissected from the surrounding tissue. Animals were sacrificed by $\mathrm{CO}_{2}$ asphyxiation, and the outflow veins were harvested for histologic or protein analyses. For histologic studies, all vessels were perfusion-fixed before removal.

\section{Histology and immunohistochemistry}

Hematoxylin and eosin (H\&E) staining was used to assess histomorphometric remodeling. Paraffinembedded sections $(4 \mu \mathrm{m})$ removed from the outflow vein in each animal at day 14 were stained with $H \& E$ and immunostained with $\alpha$-SMA (Abcam 1:200, ab32575). After mounting the slice, the capture of the co-staining images was performed by using a Nikon Eclipse $90 \mathrm{i}$ fluorescence microscope. The area was measured by tracing the vessel wall automatically. The $\alpha$-SMA indices were calculated after counting the number of positive brown cells/total cells 100 by using Image-Pro Plus 6.0. The optical density of the image was analyzed by calculating the integral optical density (IOD) value. Five non-overlapping fields of each slice were randomly selected to take the average IOD value.

\section{Immunofluorescence studies}

Fluorescein was labeled on the corresponding antibody before it reacted directly with the corresponding antigen. The PBS (0.01 mol/L, pH 7.4) was discarded after a 10 -min soaking process to preserve the humidity of the specimen. An adequately diluted, fluorescent-labeled antibody solution was added to completely submerge the specimen, and it was placed in a covered enamel box for a specific time (reference: $30 \mathrm{~min}$ ). The glass slide was taken out and put on the glass stand subsequently. It was rinsed with PBS $(0.01 \mathrm{~mol} / \mathrm{L}, \mathrm{pH} 7.4)$ and then soaked in three cylinders (over $0.01 \mathrm{~mol} / \mathrm{L}, \mathrm{pH} 7.4$ ) in order (each cylinder 3-5 min), oscillating from time to time. The glass slide was taken out and wiped with a 
filter paper to remove excess moisture. A drop of buffer glycerol was added to cover the glass before the specimen got dried. The sections were observed immediately with a fluorescence microscope.

\section{Detection of apoptotic cells}

DNA terminal transferase-mediated notch terminal labeling (TUNEL) was used to detect apoptotic cell levels in the specimens. The detection process was strictly carried out using diaminobenzidine (DAB) color reaction, according to the kit instructions.

\section{Western blot (WB) analysis}

Vein tissue extracts were prepared in RIPA buffer, and the protein concentration was measured by BCA kit (P0012S, Beyotime Biotechnology). Approximately 30 $\mu \mathrm{g}$ of protein was separated by SDS-PAGE. After being transferred to PVDF membranes, the membranes were blocked with 5\% skimmed milk and incubated with primary antibody: rabbit polyclonal antibody VEGF-A (Abcam, 1:600, ab1316), rabbit anti-EphB4 (Immunoway, 1:600, YT1587), rabbit anti-TIMP1 (Immunoway, 1:600, YT4658), rabbit anti-TIMP2 (Immunoway, 1:600, YT4659), and rabbit anti-caspase 3 (Immunoway, 1:600, ab2302), probing separately. After incubating for $1 \mathrm{~h}$ at room temperature, the membranes were incubated with the corresponding secondary antibody (Affinity, 1:5000) at room temperature for $30 \mathrm{~min}$ to wash the membrane. ECL chemiluminescence reaction (Tanon 5200 luminescence imaging system) was performed. Then, it was scanned after exposure, development, and fixation. Quantitative analysis was performed with the Image J imaging analysis system. Each of the WB assays was performed in triplicate to ensure that the interobserver variability was less than $10 \%$.

\section{Statistical analysis}

Data were expressed as mean \pm SD for continuous variables. Non-normally distributed data were described as median (range). Categorical data were expressed as percentages. Differences in categorical variables among groups were determined by the Chi-square test, with the Yates' correction or the Fisher exact probability test, as appropriate. Differences in continuous variables were determined by analysis of variance (unpaired Student's $t$-test) or non-parametric tests, as appropriate. Multiple comparisons were performed with two-way analysis of variance (ANOVA) followed by Student's $t$-test with post hoc Bonferroni's correction. Comparison between the time points was performed by one-way ANOVA followed by pairwise comparisons with $P$-value adjustment. Differences were considered statistically significant when the value of $P$-value was less than 0.05 .

\section{RESULTS}

\section{Demographic and clinical characteristics and the stenotic lesion anatomic characteristics in patients}

Patient characteristics are summarized in Table 1. The median age at the time of renal failure of the patients was 49.5 years (range from 38 to 71 years) in the case group and 51 years (range from 41 to 74 years) in the control group. The median duration from the beginning of HD was 44 (range 25-60) months in the case group. There were eight AVF cases on the left side in the case group and seven cases on the left side in the control group. The causes of ESRD in patients enrolled in this study were diabetic nephropathy (35\%), glomerulonephritis $(25 \%)$, hypertensive nephrosclerosis $(15 \%)$, polycystic kidney disease $(10 \%)$, tubulointerstitial nephritis $(5 \%)$, IgA nephropathy (5\%), and unknown disease $(5 \%)$. Comorbidities included congestive heart failure $(n=$ $7,35 \%)$, diabetes $(n=7,35 \%)$, coronary artery disease $(n=8,40 \%)$, valvular heart disease $(n=3,15 \%)$, and hypertension $(n=16,80 \%)$. Fifteen of the patients $(75 \%)$ received dialysis $>2$ times per week. Sixteen patients $(80 \%)$ received antiplatelet therapy after the AVF creation or repair operation.

The anatomical lesion characteristics are displayed in Table 2. Preoperative ultrasound revealed that the stenosis was located in the feeding artery in three patients, in the arteriovenous anastomosis in four patients, in the juxta-anastomosis vein in nine patients, in the proximal outflow vein in one patient, and in the elbow vein in one patient. The length of the stenosis was $\leq 2 \mathrm{~cm}$ in four patients and $>2 \mathrm{~cm}$ in six patients. Moderate stenosis $(\geq 50 \%$ and $<75 \%$ ) was present in one patient, severe $(\geq 75 \%$ and $<90 \%)$ stenosis in four patients, and critical stenosis $(>90 \%)$ or complete occlusion was found in five patients.

\section{Blood urea nitrogen (BUN) and serum creatinine (SCR) in the murine model after treatment}

In the murine model of this study, elevated BUN and SCR levels were observed after adenine feeding, which is similar to the typical clinical scenario. The mean BUN and SCR at baseline were $28 \pm 5$ and $0.26 \pm 0.1 \mathrm{mg} / \mathrm{dL}$, respectively. The mean BUN and SCR increased significantly at 4 weeks after adenine feeding compared with the baseline $(P<$ 0.05). Besides, there was no difference in the mean BUN and SCR among the four groups.

HEE staining showed the pattern of outflow vein
stenosis in misfunctioned AVF in HD patients
and the protective effect of anti-VEGF therapy on 
Huang et al: : Effect of local anti-VEGF therapy of the formation of stenosis in AVF

\begin{tabular}{|c|c|c|c|}
\hline \multicolumn{4}{|c|}{$\begin{array}{l}\text { Table 1: Baseline demographic and clinical characteristics } \\
\text { of the patients }\end{array}$} \\
\hline Patient characteristics & $\begin{array}{l}\text { Case group } \\
(n=10)\end{array}$ & $\begin{array}{l}\text { Control group } \\
(n=10)\end{array}$ & $P$-value \\
\hline $\begin{array}{l}\text { Age, years, median } \\
\text { (range) }\end{array}$ & $\begin{array}{l}49.5(38- \\
71)\end{array}$ & $51(41-74)$ & $0.273^{\#}$ \\
\hline \multicolumn{4}{|l|}{ Sex } \\
\hline Male & 6 & 6 & $1.00^{* *}$ \\
\hline Female & 4 & 4 & \\
\hline $\begin{array}{l}\text { AVF age, months, mediar } \\
\text { (range) }\end{array}$ & n $44(25-60)$ & 0 & $<0.001^{\#}$ \\
\hline \multicolumn{4}{|l|}{ AVF side } \\
\hline Left & 8 & 7 & $1.00^{* *}$ \\
\hline Right & 2 & 3 & \\
\hline \multicolumn{4}{|l|}{ Cause of ESRD } \\
\hline Diabetes & 3 & 4 & $0.720^{*}$ \\
\hline Glomerulonephritis & 2 & 3 & \\
\hline ADPKD & 1 & 1 & \\
\hline $\begin{array}{l}\text { Hypertensive } \\
\text { nephrosclerosis }\end{array}$ & 2 & 1 & \\
\hline Tubulointerstitial & 1 & 0 & \\
\hline IgA nephropathy & 0 & 1 & \\
\hline Unknown & 1 & 0 & \\
\hline \multicolumn{4}{|l|}{ Comorbidities } \\
\hline Congestive heart failure & 4 & 3 & $1.00^{* *}$ \\
\hline Diabetes & 4 & 3 & $1.00^{* *}$ \\
\hline Coronary artery disease & 5 & 3 & $1.00^{* *}$ \\
\hline Valvular heart disease & 1 & 2 & $1.00^{* *}$ \\
\hline Hypertension & 8 & 8 & $1.00^{* *}$ \\
\hline \multicolumn{4}{|l|}{ Dialysis frequency, /week } \\
\hline$\leq 2$ & 2 & 3 & $1.00^{* *}$ \\
\hline$>2$ & 8 & 7 & \\
\hline \multicolumn{4}{|l|}{$\begin{array}{l}\text { Antiplatelet therapy } \\
\text { (postoperation) }\end{array}$} \\
\hline Yes & 7 & 9 & $1.00^{* *}$ \\
\hline No & 3 & 1 & \\
\hline
\end{tabular}

ESRD: end-stage renal disease; ADKPD: autosomal dominant polycystic kidney disease.

${ }^{*}$ Chi-Square test; " ${ }^{*}$ Fisher exact probability test (two-tailed); ; ${ }^{*}$ nonparametric tests.

\section{vascular lumen in mouse models}

We found that the hyperplasia of cells in intima/media in the outflow vein of patients in the AVF group was significantly heavier under the light microscope than that in the control group. The proliferating cells protruding toward the lumen could be observed after H\&E staining in the AVF group. Hyperplasia of ECs, smooth muscle cells, and fibroblasts could be observed in the intima and media. The cells were arranged disorderly, accompanied by extracellular matrix deposit accumulation, neovascularization, and severe infiltration of monocytes and macrophages,

\begin{tabular}{lll}
\hline \multicolumn{3}{l}{ Table 2: Stenotic lesion anatomic characteristics } \\
\hline Position & Case group & Control group \\
\hline Inflow artery & 3 & 0 \\
Anastomosis & 3 & 0 \\
Outflow vein (juxta-anastomosis) & 9 & 0 \\
Outflow vein (proximal) & 1 & 0 \\
Elbow vein & 1 & 0 \\
Length (cm) & & \\
$\leq 2$ & 4 & 0 \\
$>2$ & 6 & 0 \\
Stenosis grade (\%) & & 0 \\
$\geq 50$ and $<75$ & 1 & 0 \\
$\geq 75$ and $<90$ & 4 & 0 \\
$\geq 90$ or complete occlusion & 5 & \\
\hline
\end{tabular}

especially in the inner membrane. On the contrary, in the control group, the intima of the lesion was composed of a single layer of ECs. Also, the middle membrane was composed of three to four layers of smooth muscle cells, which were neatly arranged without thickening, new blood vessel formation, or inflammatory cell infiltration in intima and media. The extracellular matrix was standard (Figure 1A). Histomorphometric remodeling after 14 days was also assessed by observing and comparing H\&E-stained tissues from different groups. The mean lumen volume was more significant in the Avastin or the LV-shRNAVEGF-A group compared with that of the control-shRNA group (Figure 1B) on the 14th day after AVF operation. The intimal thickness of Avastin vessels was significantly lesser than that of the control-shRNA group $(P<0.05$, Figure 1C).

\section{VEGF-A expression was lower in Avastin-treated vessels}

To determine the relationship of VEGF-A expression and the proliferation of cells, we performed immunofluorescence studies and WB on sections from the outflow vein of the patients' specimens. VEGF was more highly expressed in the AVF group (Figure 2A, B). In the mice experiments, the expression of VEGF-A in the control-shRNA group was significantly higher than that of the sham group on day $14(P<0.05$, Figure 2C). VEGF-A expression was lower in the Avastin group than in the control-shRNA group $(P<$ 0.05, Figure 2C). Ephb4, which is Ephrin b2 receptor, and VEGF are both VEGFs and can promote cell proliferation. The mean levels of Ephb4 protein in the outflow vein of the LV-shRNA-VEGF-A group and Avastin group were significantly lower compared with those in the controlshRNA group on day 14 ( $P<0.05$, Figure $2 \mathrm{C})$. 

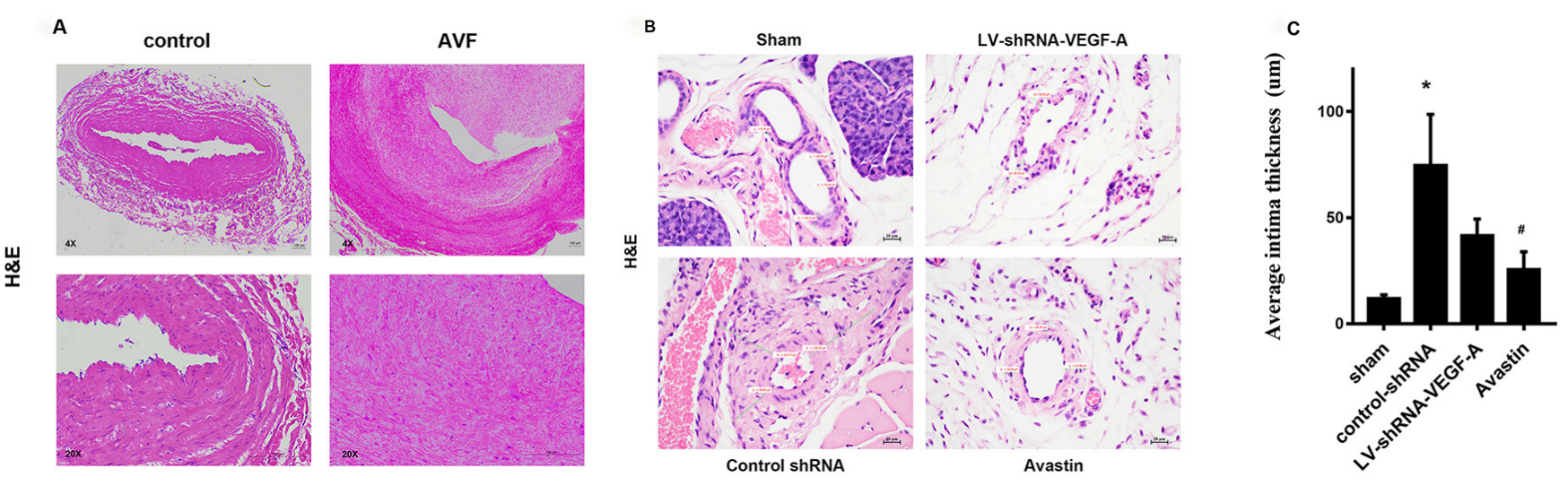

Figure 1: (A) H\&E-stained tissues from patients in control and AVF groups: intima/media in the AVF group were significantly thicker. (B and C) Four mouse model groups: sham group, control-shRNA group, LV-shRNA-VEGF-A group, Avastin group. The thickness of intima was more evident in the control-shRNA group compared with the sham group $(P<0.05)$. The intimal thickness of Avastin-treated vessels was lesser and the mean lumen area was significantly larger compared with the control-shRNA vessels on day $14(P<0.05)$.

*Indicates a significant difference compared with the sham group $(P<0.05)$.

\#Indicates a significant difference compared with the control-shRNA group $(P<0.05)$.

Values are shown as means \pm SD from three independent experiments. Representative images are shown from a total of four animals per group.

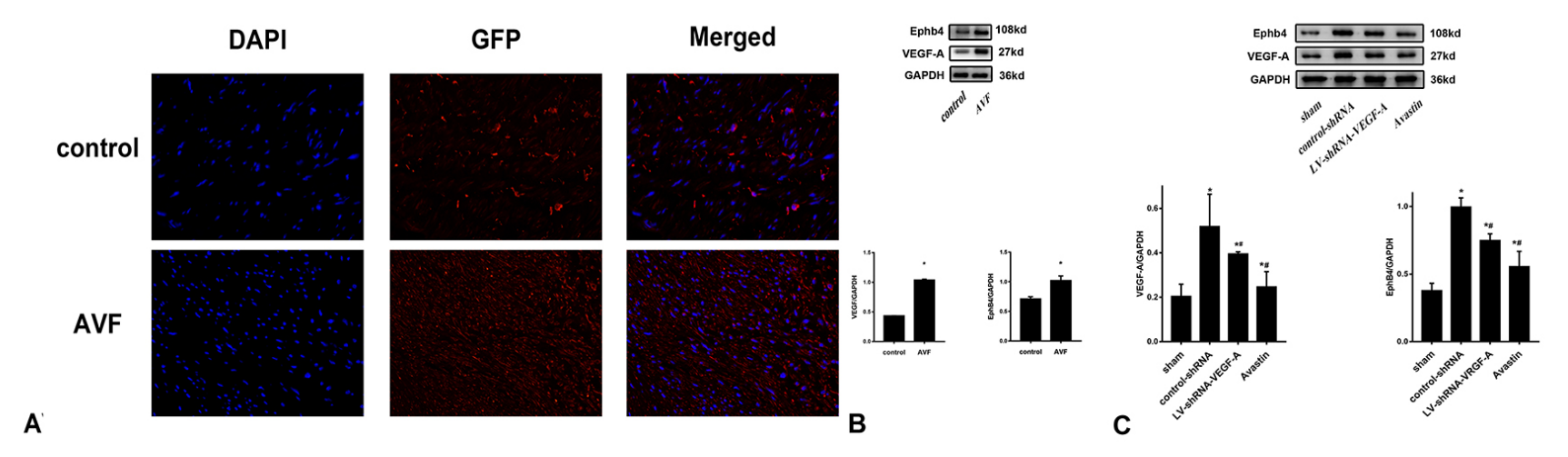

Figure 2: (A) The images under confocal microscopy in immunofluorescence studies of representative sections of tissues from the outflow vein in patients of stenosis and control groups. The first column shows DAPI intensity indicating blue-stained nuclei. The second column shows GFP intensity. Magenta-stained cells are GFP positive. The third column depicts merged images. These sections demonstrate different levels of expression of the VEGF protein in the outflow vein between the two groups. (B) The expression of vascular tissue VEGF-A and Ephb4 protein levels in patients. VEGF and Ephb4 were more highly expressed in the AVF group $(P<0.05)$. (C) Expression of VEGF-A and Ephb4 in mouse models. Compared to the sham group, the level of expression of VEGF-A and Ephb4 in the control-shRNA group was significantly higher $(P<0.05)$. VEGF-A expression in the Avastin group was lower than in the control-shRNA group $(P<0.05)$, and the mean levels of Ephb4 protein in the outflow vein of the LV-shRNA-VEGF-A group and Avastin group were significantly lower $(P<0.05)$.

"Indicates a significant difference compared with the sham group $(P<0.05)$.

\#Indicates a significant difference compared with the control-shRNA group $(P<0.05)$.

Values are shown as means \pm SD from three independent experiments. Representative images are shown from a total of four animals per group.

\section{Adventitial injection of Avastin was associated with high expression of apoptotic markers on day 14}

Apoptosis was first assessed by TUNEL staining performed on tissue obtained from the intraoperative specimen of the patients. There was an apparent difference between the two groups (Figure 3A). The average activity of the apoptosis executioner caspase 3 was significantly lower in patients of the control groups $(P<0.05$, Figure $3 \mathrm{~B})$. The same tendency toward low expression was observed in mice in the Avastin-treated vessels, when compared with the control-shRNA group (Figure 3C).

\section{Cellular proliferation in Avastin-treated outflow vein was less significant}

Several studies have shown increased expression of MMP2 and MMP9 in the tissues of misfunctioned AVF and graft in animal models and clinical samples. We focus on the bioinformatics network of interaction among VEGF-A and its related regulated MMP2, TIMP1, TIMP2 and EphB4 molecules by analyzing the STRING PPI database (Figure 4A). Based on the test results of samples in animal and patient studies, we found that the reduction of VEGF-A could downregulate related molecules and subsequently reduce intimal hyperplasia and abnormal 
A
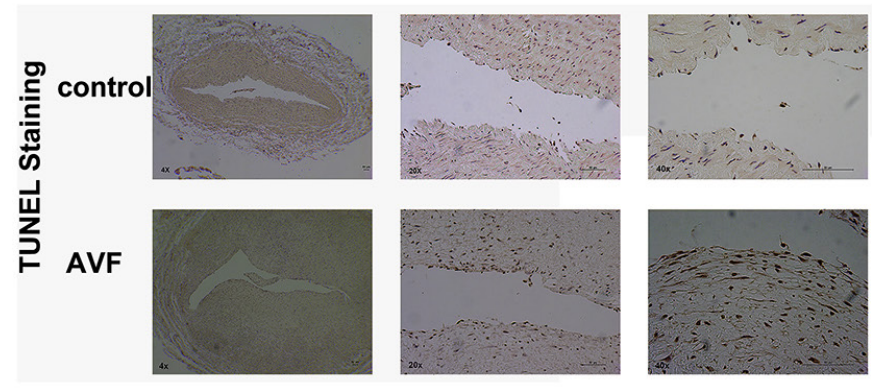

Figure 3: (A) A representative section from TUNEL staining at the venous stenosis of patients. Nuclei staining brown are positive for TUNEL. The negative control is shown where the recombinant terminal deoxynucleotidyl transferase enzyme was omitted. Scale bar is $50 \mu \mathrm{M}$. (B) Caspase 3 in patients. Caspase 3 was significantly lowered in the control groups of patients $(P<0.05)$. (C) The expression of the Caspase 3 protein levels of vascular tissue in the four mice groups. The same tendency toward low expression was observed in Avastin-treated vessels compared with the control-shRNA group $(P<0.05)$.

"Indicates a significant difference compared with the sham group $(P<0.05)$.

\#Indicates a significant difference compared with control-shRNA group $(P<0.05)$.

Values are shown as means \pm SD from three independent experiments. Representative images are shown from a total of four animals per group.

A

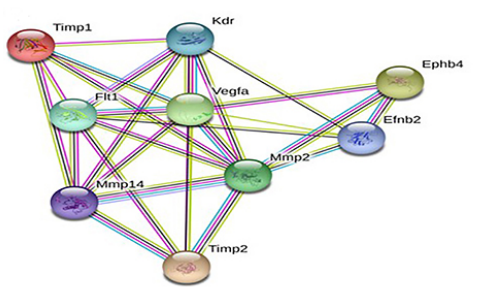

B

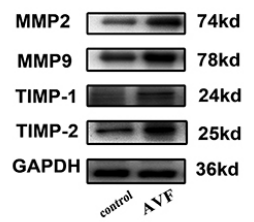

C

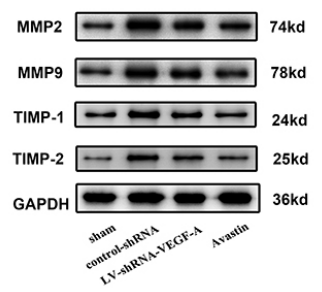

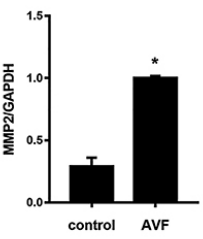
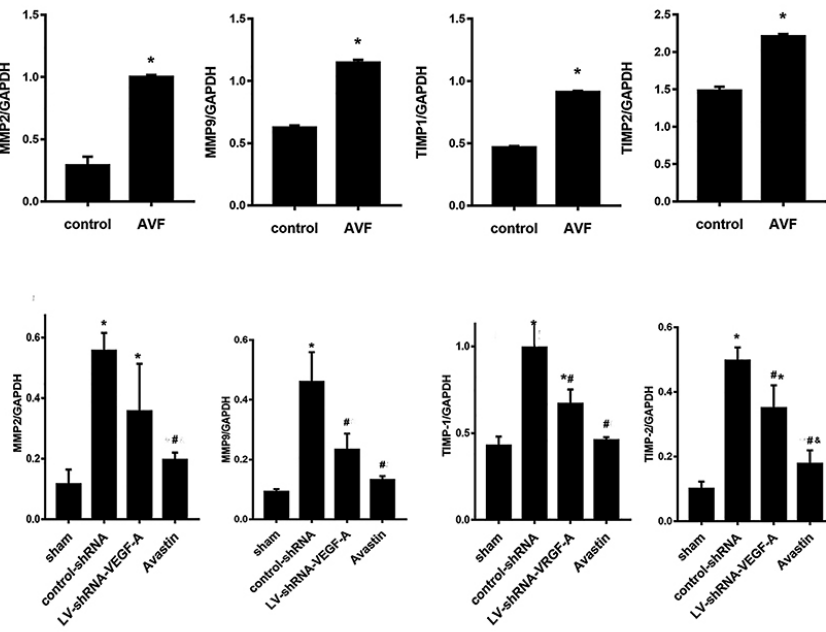
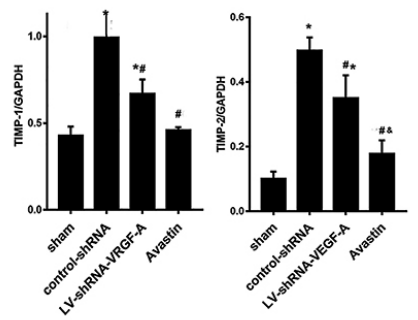

Figure 4: (A) Bioinformatics network of interaction among VEGF-A and its downstream-regulated molecules MMP2, TIMP1, TIMP2, and EphB4 by analyzing STRING PPI database. (B) MMP2, MMP9, TIMP1, and TIMP2 protein expression levels in the tissue of outflow veins in patients of different groups. MMP2, MMP9, TIMP1, and TIMP2 were higher in the AVF groups. (C) MMP2, MMP9, TIMP1, and TIMP2 in the four mice groups. The level of MMP9 expression was lower in the Avastin and LV-shRNA groups compared with the control-shRNA group on day $14(P<0.05)$, and the level of MMP2 expression was lower in the Avastin group compared with the control-shRNA group $(P<0.05)$. The expression of TIMP1 and TIMP2 in the outflow vein was significantly lower in the Avastin-treated group and LV-shRNA-transduced group compared with the control-shRNA group $(P<0.05)$. Also, there was a substantial difference in TIMP2 expression between Avastin-treated and LV-shRNA groups $(P<0.05)$.

*Indicates a significant difference compared with the sham group $(P<0.05)$.

\#Indicates a significant difference compared with the control-shRNA group $(P<0.05)$.

Eindicates a significant difference compared with the LV-shRNA-VEGF group.

Values are shown as means \pm SD from three independent experiments. Representative images are shown from a total of four animals per group.

vascular remodeling. This discovery would be promising to improve the long-term patency in favor of the stability of HD treatment. The results are consistent with the results obtained by us from experiments on clinical samples (Figure 4B). MMP9 expression was lower in outflow veins in the Avastin and LV-shRNA-VEGF-A groups, compared to that of the control-shRNA group $(P<0.05)$; likewise, MMP2 expression was lower in the Avastin group than in the control-shRNA group on day $14(P<0.05$, Figure 4C). The expression of TIMP1 and TIMP2 in the outflow vein was significantly lower in the Avastin group and LV-shRNA-VEGF-A group, compared with the controlshRNA group $(P<0.05)$. There was a substantial difference in TIMP2 expression between the Avastin and LV-shRNAVEGF-A groups ( $P<0.05$, Figure $4 C)$. 


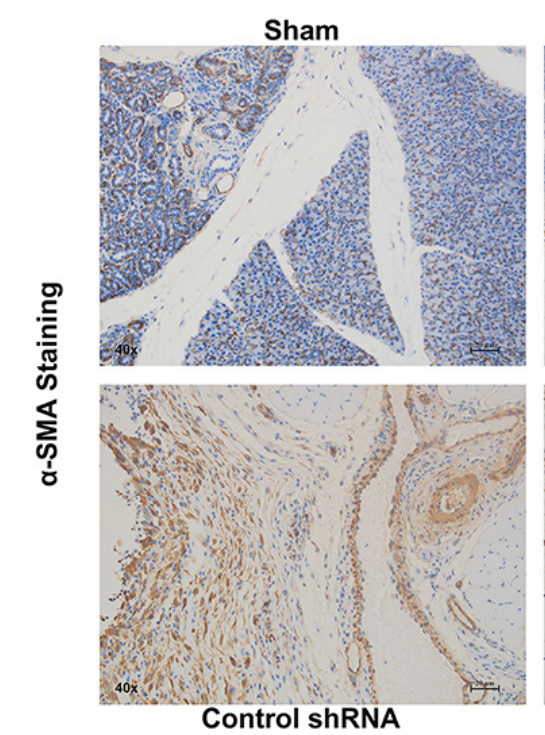

A
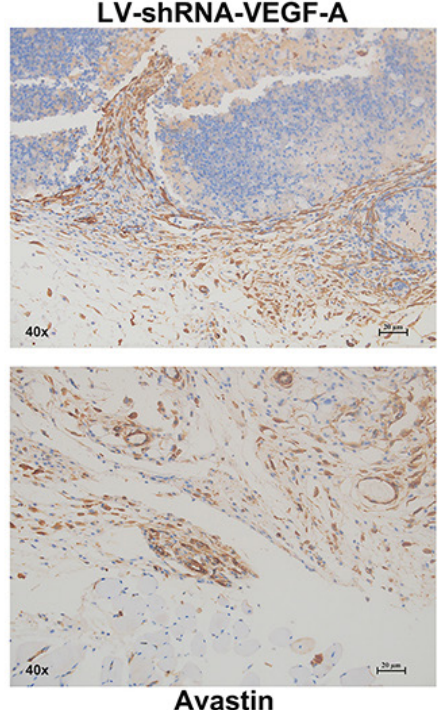

Avastin

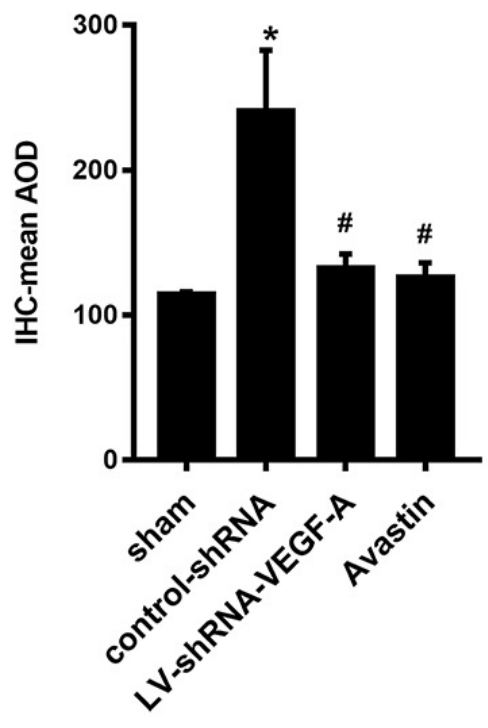

B

Figure 5: (A) Expression of vascular tissue $\alpha$-SMA levels in four groups of mouse models. The average $\alpha$-SMA expression at the outflow vein of the Avastin and LV-shRNA-VEGF-A groups was significantly lower than the expression in the control-shRNA group on day 14. (B) IHC-mean AOD: In the meantime, the $\alpha$-SMA index was significantly lower in the LV-shRNA-VEGF-A and Avastin groups compared with that in the control-shRNA group $(P<0.05)$.

"Indicates a significant difference compared with the sham group $(P<0.05)$.

${ }^{\#}$ Indicates a significant difference compared with the control-shRNA group $(P<0.05)$.

Values are shown as means \pm SD from three independent experiments. Representative images are shown from a total of four animals per group.

\section{Adventitial transduction of LV-shRNA-VEGF-A and Avastin injection to the outflow vein decrease a-SMA expression}

The majority of the cells comprising VNH are positive for $\alpha$-SMA. The outflow vein in the LV-shRNA-VEGF-A group and the control group was stained with IHC methods, targeting $\alpha$-SMA. The average $\alpha$-SMA expression in the outflow vein of the Avastin group and LV-shRNAVEGF-A group was significantly lower than that of the control-shRNA group on day 14 (Figure 5A). In the meantime, the $\alpha$-SMA index was significantly lower in the LV-shRNA-VEGF-A and Avastin groups, compared to that in the control-shRNA group $(P<0.05$, Figure $5 \mathrm{~B})$.

\section{DISCUSSION}

The number of ESRD patients receiving HD therapy is projected to double in the next decade. ${ }^{[28,29]}$ Long-term stability and patency of vascular access are critical to ESRD patients undergoing HD. VNH induces venous stenosis formation, leading to dysfunction of AVF. In recent studies, local, sustained release of drugs in AVF vessels was found to inhibit the proliferation of smooth muscle cells and myofibroblasts. Thus, dysregulation of the VNH pathway was mitigated. ${ }^{[30,31]}$ However, while inhibiting the proliferation of smooth muscle cells, they also delay the repair of the damage of ECs and the process of re-endothelialization. High concentrations of Avastin or transduced LV-shRNA-VEGF-A in local tissues would inhibit proliferation and promote apoptosis. ${ }^{[32]}$ Some animal studies have confirmed this conclusion. ${ }^{[14,33]}$ In our experiment in the murine model, the proliferation markers in the Avastin group were lower than those in the control group, as expected. In contrast to previous studies, however, the apoptotic markers in the Avastin group were significantly lower than those in the control group in our study. The possible explanation is as follows. In the study of Nirvana, ${ }^{[7]}$ pathologic changes such as cell proliferation and migration in the intima/medial layer of the outflow vein had already occurred in the early stage. The Avastin injected afterward inhibited neovascularization, and the imbalance between the proliferated tissue and neovascularization resulted in apoptosis. However, in our study, due to the early application of Avastin, endothelial proliferation was initially blocked. There was less excessive cell proliferation. Therefore, fewer hyperproliferative cells led to less apoptosis and less expression of apoptotic markers in the subsequent pathophysiologic process.

VEGF-A is a multifunctional cytokine. It plays crucial roles in the physiological functions and regulatory processes involved in developing, such as proliferation, migration, and death of cells. ${ }^{[19,34]}$ Gh found that VEGF-A can promote neovascularization mediation and re-endothelialization of damaged wall intima, in addition to smooth muscle 
cell migration. ${ }^{[35]}$ Yet, the specific mechanism of VEGF in the formation of $\mathrm{VNH}$ is unknown. In the previous studies, a high concentration of VNH formation markers, VEGF-A and VEGFR-1, can be detected in the venous stenosis lesion in animal models and in adventitia and neointima in dysfunctional AVF and graft in HD patients. ${ }^{[36,37]}$ The results were the same as ours. VEGF-A was highly expressed in AVF patients and control-shRNA-transduced mice. Conversely, the VEGF-A expression was lower in the Avastin group.

An end-to-end AVF on the murine model was constructed in previous studies ${ }^{[38,39]}$ to determine the pathologic mechanism of outflow vein stenosis. After that, Cartier introduced a new method that the end of the common carotid artery could be connected to the side of the external jugular vein. ${ }^{[40,41]}$ However, AVFs are usually constructed by anastomosing the end of the vein to the side of an artery in HD patients. The configuration of the AVF is crucial for determining the profile of hemodynamic factors contributing to endothelial dysfunction and subsequent development of neointimal hyperplasia. ${ }^{[42,43]}$ So, the latter procedure to simulate the hemodynamic state of the end-side anastomosis to the maximum extent was not needed. We used a novel murine model of stenosis of AVF outflow vein in which the AVF configuration resembled hemodynamics in situ (AVF with venous-end to the arterial-side anastomosis of the forearm in HD patients).

MMP2 and MMP9 in the MMPS family could degrade the extracellular matrix, and they are necessary for the migration of damaged epicardial and mesial cells to the neointima. Hydrolysates of MMPS could induce neovascularization, stenosis, ${ }^{[12,44]}$ and extracellular matrixinduced angiogenesis of the outflow vein. Recent studies have also confirmed that endometrial injury can upregulate MMP2 expression and promote SMCs' migration, ${ }^{[45]}$ leading to thickening of the intima. ${ }^{[4]}$ The results are consistent with the results of our experiments on clinical samples. We found that MMP9 expression was lower in vessels in the Avastin and LV-shRNA groups, compared to that in the control-shRNA group $(P<0.05$, respectively). Meanwhile, MMP2 expression was lower in the Avastin group than in the control-shRNA group on day 14 (Figure 5). MMP levels are negatively regulated physiologically by their inhibitor TIMPs and $\alpha 2$-macroglobulin. TIMP2 can inhibit the endothelial proliferation induced by bFGF. ${ }^{[4]}$ Moreover, TIMP1 and TIMP2 have antiapoptotic properties. ${ }^{[48,49]}$ But the results of our research are not consistent with those of previous studies. The possible reasons are: (1) the observation time might not be long enough and (2) the effect on the inhibition of TIMP1 and TIMP2 was not reflected.
When intimal hyperplasia occurs, the SMCs in the central membrane proliferate and migrate to the intima, forming a thickened new intima. This series of changes exacerbate vascular stenosis. Moreover, a variety of factors, such as a uremic toxin, inflammation, oxidative stress, and local hemodynamics, ${ }^{[50]}$ cause dysfunction of vascular ECs and endothelial disintegration of AVF in HD patients. ${ }^{[51]}$ Continuous stimulation induces dysregulation of cellular compensatory function. As the integrity and function of the endothelium are destroyed, ${ }^{[52]}$ a variety of inflammatory factors and cytokines are released, leading to a series of subsequent pathophysiologic processes such as excessive cell proliferation, massive deposition of the extracellular matrix, and intimal hyperplasia. ${ }^{[53]}$

The transmembrane receptor tyrosine kinase family member, EphB4, is a determinant of venous formation during embryonic development. We verified the theory that EphB4 inhibits thickening of the neointima of veins. ${ }^{[54]}$ In our experiments, the expression of EphB4 protein was lower in Avastin and LV-shRNA-VEGF-A groups compared with the control-shRNA group (Figure 3).

In our present study, the effect of Avastin on declining the expression of VEGF-A, MMP2 and TIMP2 and promoting apoptosis is more pronounced than that of LV-shRNA-VEGF-A gene silencing. The whole network system activated by Avastin led to a series of changes such as decrease of cell proliferation, decrease of proliferation of $\alpha$-SMA-positive cells, increase of apoptosis, decrease of intimal thickness, decrease of stenosis, and increase of positive vascular remodeling. The clinical value of this study is that it demonstrates the pathophysiologic mechanisms and practical efficacy of anti-VEGF therapy in autologous AVF operation for the prevention of outflow vein stenosis.

\section{CONCLUSION}

In this study, VNH was found as a critical factor that could facilitate HD vascular access failure. Meanwhile, VEGF expression is significantly increased in vascular ECs in a stenosed or occluded outflow vein of dysfunctional AVF. Such pathophysiologic processes may undermine the function of AVF in HD patients. Consequently, local injection of Avastin into the adventitia of the proximal outflow vein in autologous AVF procedure has excellent potential to prevent the subsequent local stenosis of the proximal outflow vein.

\section{Ethics approval and consent to participate}

The ethics committee of The First Hospital of China Medical University approved the protocol (Ethical Lot Number: AF-SOP-07-1,1-01). Written informed consent was obtained from each patient or from appropriate 
surrogates for the patients unable to give consent.

\section{Consent for publication}

Not applicable. No individual personal data are included in the study.

\section{Availability of data and materials}

The datasets generated and analyzed during this study are not publicly available, owing to currently ongoing research studies, but the data are available from the corresponding author on reasonable request.

\section{Competing interests}

The authors declare that they have no competing interests.

\section{Source of Funding}

This work was supported by the following funds: (1) Natural Fund Guidance Plan (82070763;81770766), (2) Liaoning Provincial Natural Fund Guidance Plan (2019-ZD-0426), (3) Special Fund for Clinical Medicine Research of Chinese Medical Association (20010040796), (4) Liaoning Province Key R\&D Guidance Project Plan (2020JH2/10300045;2019JH8/10300020), and (5) Shenyang Science and Technology Plan Population and Health Special Project (19-112-4-031). The funders had no role in study design, data collection, analysis, decision to publish, or manuscript preparation.

\section{Acknowledgments}

The authors would like to thank all the doctors, nurses, technicians, and patients involved in this study for their dedication to the study.

\section{REFERENCE}

1. Wong CY, de Vries MR, Wang Y, van der Vorst JR, Vahrmeijer AL, van Zonneveld AJ, et al. Vascular remodeling and intimal hyperplasia in a novel murine model of arteriovenous fistula failure. J Vasc Surg 2014;59:192-201 el.

2. Zouaghi MK, Lammouchi MA, Hassan M, Rais L, Krid M, Smaoui W, et al. Determinants of patency of arteriovenous fistula in hemodialysis patients. Saudi J Kidney Dis Transpl 2018;29:615-22.

3. Falk A. Maintenance and salvage of arteriovenous fistulas. J Vasc Interv Radiol 2006;17:807-13.

4. US Renal Data System 2019 Annual Data Report: Epidemiology of Kidney Disease in the United States. Am J Kidney Dis 2019;S02726386(19)31008-X.

5. Lee T. Novel paradigms for dialysis vascular access: downstream vascular biology--is there a final common pathway? Clin J Am Soc Nephrol 2013;8:2194-201.

6. Jia L, Wang L, Wei F, Li C, Wang Z, Yu H, et al. Effects of Caveolin-1ERK1/2 pathway on endothelial cells and smooth muscle cells under shear stress. Exp Biol Med (Maywood) 2020;245:21-33.

7. Sadaghianloo N, Contenti J, Dardik A, Mazure NM. Role of Hypoxia and Metabolism in the Development of Neointimal Hyperplasia in Arteriovenous Fistulas. Int J Mol Sci 2019;20:5387.

8. Sadaghianloo N, Contenti J, Declemy S, Ambrosetti D, Zdralevic M, Tannour-Louet M, et al. Hypoxia and hypoxia-inducible factors promote the development of neointimal hyperplasia in arteriovenous fistula. J Physiol 2021; 599:2299-321.

9. Remuzzi A, Ene-Iordache B. Novel paradigms for dialysis vascular access: upstream hemodynamics and vascular remodeling in dialysis access stenosis. Clin J Am Soc Nephrol 2013;8:2186-93.

10. Cai C, Kilari S, Singh AK, Zhao C, Simeon ML, Misra A, et al. Differences in Transforming Growth Factor- $\beta 1 /$ BMP7 Signaling and Venous Fibrosis Contribute to Female Sex Differences in Arteriovenous Fistulas. J Am Heart Assoc 2020;9:e017420.

11. Wang Y, Krishnamoorthy M, Banerjee R, Zhang J, Rudich S, Holland $\mathrm{C}$, et al. Venous stenosis in a pig arteriovenous fistula model--anatomy, mechanisms and cellular phenotypes. Nephrol Dial Transplant 2008;23:525-33.

12. Janardhanan R, Yang B, Vohra P, Roy B, Withers S, Bhattacharya S, et al. Simvastatin reduces venous stenosis formation in a murine hemodialysis vascular access model. Kidney Int 2013;84:338-52.

13. Roy-Chaudhury P, Wang Y, Krishnamoorthy M, Zhang J, Banerjee R, Munda R, et al. Cellular phenotypes in human stenotic lesions from haemodialysis vascular access. Nephrol Dial Transplant 2009;24:2786-91.

14. Yang B, Janardhanan R, Vohra P, Greene EL, Bhattacharya S, Withers $\mathrm{S}$, et al. Adventitial transduction of lentivirus-shRNA-VEGF-A in arteriovenous fistula reduces venous stenosis formation. Kidney Int 2014;85:289-306.

15. Zhao C, Zuckerman ST, Cai C, Kilari S, Singh A, Simeon M, et al. Periadventitial Delivery of Simvastatin-Loaded Microparticles Attenuate Venous Neointimal Hyperplasia Associated With Arteriovenous Fistula. J Am Heart Assoc 2020;9:e018418.

16. Croatt AJ, Grande JP, Hernandez MC, Ackerman AW, Katusic ZS, Nath KA. Characterization of a model of an arteriovenous fistula in the rat: the effect of L-NAME. Am J Pathol 2010;176:2530-41.

17. Liang M, Woodard LE, Liang A, Luo J, Wilson MH, Mitch WE, et al. Protective role of insulin-like growth factor-1 receptor in endothelial cells against unilateral ureteral obstruction-induced renal fibrosis. Am J Pathol 2015;185:1234-50.

18. Yang L, Zhang D, Wu H, Xie S, Zhang M, Zhang B, et al. Basic Fibroblast Growth Factor Influences Epidermal Homeostasis of Living Skin Equivalents through Affecting Fibroblast Phenotypes and Functions. Skin Pharmacol Physiol 2018;31:229-37.

19. Melincovici CS, Boşca AB, Şuşman S, Mărginean M, Mihu C, Istrate M, et al. Vascular endothelial growth factor (VEGF) - key factor in normal and pathological angiogenesis. Rom J Morphol Embryol 2018;59:455-67.

20. Siveen KS, Prabhu K, Krishnankutty R, Kuttikrishnan S, Tsakou M, Alali FQ, et al. Vascular Endothelial Growth Factor (VEGF) Signaling in Tumour Vascularization: Potential and Challenges. Curr Vasc Pharmacol 2017;15:339-51.

21. Hegde PS, Wallin JJ, Mancao C. Predictive markers of anti-VEGF and emerging role of angiogenesis inhibitors as immunotherapeutics. Semin Cancer Biol 2018;52:117-24.

22. Wågsäter D, Zhu C, Björkegren J, Skogsberg J, Eriksson P. MMP-2 and MMP-9 are prominent matrix metalloproteinases during atherosclerosis development in the Ldlr(-/-)Apob(100/100) mouse. Int J Mol Med 2011;28:247-53.

23. Yakala GK, Cabrera-Fuentes HA, Crespo-Avilan GE, Rattanasopa C, Burlacu A, George BL, et al. FURIN Inhibition Reduces Vascular Remodeling and Atherosclerotic Lesion Progression in Mice. Arterioscler Thromb Vasc Biol 2019;39:387-401.

24. Erratum Regarding "KDOQI Clinical Practice Guideline for Vascular Access: 2019 Update” (Am J Kidney Dis. 2020;75[4][suppl 2]:S1-S164). Am J Kidney Dis 2021;77:551.

25. Wo K, Morrison BJ, Harada RN. Developing Duplex Ultrasound Criteria for Diagnosis of Arteriovenous Fistula Stenosis. Ann Vasc Surg 2017;38:99-104.

26. Benedetto FA, Mallamaci F, Tripepi G, Zoccali C. Prognostic value of ultrasonographic measurement of carotid intima media thickness in 
dialysis patients. J Am Soc Nephrol 2001;12:2458-64.

27. Steiner K, Ramanarayanan S, Metcalfe M, Jeevaratnum P, Selvakumar S, Narula A. Ultrasound appearances and histological correlation of native arteriovenous fistula stenoses - A retrospective case series. Semin Dial 2021;34:224-8.

28. Collins AJ, Kasiske B, Herzog C, Chen SC, Everson S, Constantini E, et al. Excerpts from the United States Renal Data System 2003 Annual Data Report: atlas of end-stage renal disease in the United States. Am J Kidney Dis 2003;42:A5-7, s1-230

29. Reid C, Seymour J, Jones C. A Thematic Synthesis of the Experiences of Adults Living with Hemodialysis. Clin J Am Soc Nephrol 2016;11:120618.

30. Masaki T, Rathi R, Zentner G, Leypoldt JK, Mohammad SF, Burns GL, et al. Inhibition of neointimal hyperplasia in vascular grafts by sustained perivascular delivery of paclitaxel. Kidney Int 2004;66:2061-9.

31. Melhem M, Kelly B, Zhang J, Kasting G, Li J, Davis H, et al. Development of a local perivascular paclitaxel delivery system for hemodialysis vascular access dysfunction: polymer preparation and in vitro activity. Blood Purif 2006;24:289-98.

32. Janardhanan R, Yang B, Kilari S, Leof EB, Mukhopadhyay D, Misra S. The Role of Repeat Administration of Adventitial Delivery of LentivirusshRNA-Vegf-A in Arteriovenous Fistula to Prevent Venous Stenosis Formation. J Vasc Interv Radiol 2016;27:576-83.

33. Nieves Torres EC, Yang B, Roy B, Janardhanan R, Brahmbhatt A, Leof E, et al. Adventitial delivery of lentivirus-shRNA-ADAMTS-1 reduces venous stenosis formation in arteriovenous fistula. PLoS One 2014;9:e94510.

34. Duque JC, Vazquez-Padron RI. Myofibroblasts: the ideal target to prevent arteriovenous fistula failure? Kidney Int 2014;85:234-6.

35. Gh K, Mhs M, H R, M D, L H, M N. Primary patency rate of native AV fistula: long term follow up. Int J Clin Exp Med 2012;5:173-8.

36. Misra S, Fu AA, Rajan DK, Juncos LA, McKusick MA, Bjarnason H, et al. Expression of hypoxia inducible factor- 1 alpha, macrophage migration inhibition factor, matrix metalloproteinase- 2 and -9 , and their inhibitors in hemodialysis grafts and arteriovenous fistulas. J Vasc Interv Radiol 2008;19:252-9.

37. Misra S, Shergill U, Yang B, Janardhanan R, Misra KD. Increased expression of HIF-1alpha, VEGF-A and its receptors, MMP-2, TIMP-1, and ADAMTS- 1 at the venous stenosis of arteriovenous fistula in a mouse model with renal insufficiency. J Vasc Interv Radiol 2010;21:1255-61.

38. Kwei S, Stavrakis G, Takahas M, Taylor G, Folkman MJ, Gimbrone MA, Jr., et al. Early adaptive responses of the vascular wall during venous arterialization in mice. Am J Pathol 2004;164:81-9.

39. Yang B, Shergill U, Fu AA, Knudsen B, Misra S. The mouse arteriovenous fistula model. J Vasc Interv Radiol 2009;20:946-50.

40. Castier Y, Lehoux S, Hu Y, Foteinos G, Tedgui A, Xu Q. Characterization of neointima lesions associated with arteriovenous fistulas in a mouse model. Kidney Int 2006;70:315-20.
41. Kang L, Yamada S, Hernandez MC, Croatt AJ, Grande JP, Juncos JP, et al. Regional and systemic hemodynamic responses following the creation of a murine arteriovenous fistula. Am J Physiol Renal Physiol 2011;301:F845-51.

42. Duque JC, Martinez L, Tabbara M, Parikh P, Paez A, Selman G, et al. Vascularization of the arteriovenous fistula wall and association with maturation outcomes. J Vasc Access 2020;21:161-8.

43. Satish M, Gunasekar P, Agrawal DK. Pro-inflammatory and proresolving mechanisms in the immunopathology of arteriovenous fistula maturation. Expert Rev Cardiovasc Ther 2019;17:369-76.

44. Janardhanan R, Kilari S, Leof EB, Misra S. Hyperglycemia-Induced Modulation of the Physiognomy and Angiogenic Potential of Fibroblasts Mediated by Matrix Metalloproteinase-2: Implications for Venous Stenosis Formation Associated with Hemodialysis Vascular Access in Diabetic Milieu. J Vasc Res 2015;52:334-46.

45. Misra S, Doherty MG, Woodrum D, Homburger J, Mandrekar JN, Elkouri $\mathrm{S}$, et al. Adventitial remodeling with increased matrix metalloproteinase-2 activity in a porcine arteriovenous polytetrafluoroethylene grafts. Kidney Int 2005;68:2890-900.

46. Ghosh A, Pechota LV, Upchurch GR, Jr., Eliason JL. Cross-talk between macrophages, smooth muscle cells, and endothelial cells in response to cigarette smoke: the effects on MMP2 and 9. Mol Cell Biochem 2015;410:75-84.

47. Lipka D, Boratyński J. [Metalloproteinases. Structure and function]. Postepy Hig Med Dosw (Online) 2008;62:328-36.

48. Groblewska M, Tycińska A, Mroczko B, Musiał W, Szmitkowski M. The role of matrix metalloproteinases in cardiovascular diseases. Pol Merkur Lekarski 2011;30:235-40.

49. Fink K, Boratyński J. The role of metalloproteinases in modification of extracellular matrix in invasive tumor growth, metastasis and angiogenesis. Postepy Hig Med Dosw (Online) 2012;66:609-28.

50. Fitts MK, Pike DB, Anderson K, Shiu YT. Hemodynamic Shear Stress and Endothelial Dysfunction in Hemodialysis Access. Open Urol Nephrol J 2014;7:33-44.

51. Brahmbhatt A, Remuzzi A, Franzoni M, Misra S. The molecular mechanisms of hemodialysis vascular access failure. Kidney Int 2016;89:303-16.

52. Dember LM, Imrey PB, Beck GJ, Cheung AK, Himmelfarb J, Huber TS, et al. Objectives and design of the hemodialysis fistula maturation study. Am J Kidney Dis 2014;63:104-12.

53. Alshanwani AR, Riches-Suman K, O'Regan DJ, Wood IC, Turner NA, Porter KE. MicroRNA-21 drives the switch to a synthetic phenotype in human saphenous vein smooth muscle cells. IUBMB Life 2018;70:649-57.

54. Muto A, Yi T, Harrison KD, Dávalos A, Fancher TT, Ziegler KR, et al. Eph-B4 prevents venous adaptive remodeling in the adult arterial environment. J Exp Med 2011;208:561-75.

How to cite this article: Huang X, Guan J, Sheng Z, Wang M, Xu T, Guo $\mathrm{G}$, et al. Effect of local anti-vascular endothelial growth factor therapy to prevent the formation of stenosis in outflow vein in arteriovenous fistula. J Transl Intern Med 2021; 9: 307-17. 